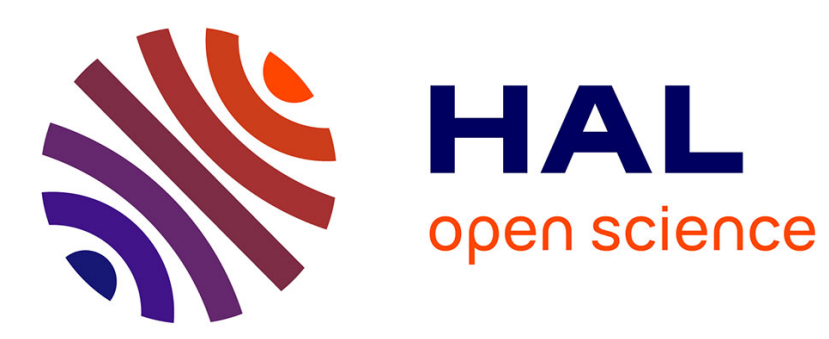

\title{
The diffusive limit of Carleman-type models in the range of very fast diffusion equations \\ Francesco Salvarani, Giuseppe Toscani
}

\section{To cite this version:}

Francesco Salvarani, Giuseppe Toscani. The diffusive limit of Carleman-type models in the range of very fast diffusion equations. 2008. hal-00272382v2

\section{HAL Id: hal-00272382 \\ https://hal.science/hal-00272382v2}

Preprint submitted on 24 Oct 2008

HAL is a multi-disciplinary open access archive for the deposit and dissemination of scientific research documents, whether they are published or not. The documents may come from teaching and research institutions in France or abroad, or from public or private research centers.
L'archive ouverte pluridisciplinaire HAL, est destinée au dépôt et à la diffusion de documents scientifiques de niveau recherche, publiés ou non, émanant des établissements d'enseignement et de recherche français ou étrangers, des laboratoires publics ou privés. 


\title{
The diffusive limit of Carleman-type models in the range of very fast diffusion equations.
}

\author{
Francesco Salvarani, Giuseppe Toscani*
}

October 24, 2008

\begin{abstract}
We improve the existing results on the limiting behavior of the Cauchy problem for a class of Carleman-like models with power-type interaction rate in the diffusive scaling with data in the spaces $L^{p}, 1 \leq p \leq \infty$. The convergence result, which has been carefully established before for exponents of the interaction rate $\alpha \leq 1$, is extended here to the range of exponents $1<\alpha<4 / 3$. In addition, we discuss the problem of establishing a good theory in the still remaining range $\alpha \in[4 / 3,2)$, by introducing a modified kinetic system which admits an explicit self-similar solution. The analysis of this solution clarifies the role of the exponent $\bar{\alpha}=4 / 3$.
\end{abstract}

\section{Introduction}

We consider a class of a one-dimensional models for a gas composed of two kinds of particles moving parallel to the $x$-axis with constant and equal speeds, of modulus $c>0$, one in the positive $x$-direction with density $u(t, x)$, the other in the negative $x$-direction with density $v(t, x)$. A general version of such a model has the following form:

$$
\left\{\begin{array}{l}
\frac{\partial u}{\partial t}+c \frac{\partial u}{\partial x}=k(u, v, x)(v-u) \\
\frac{\partial v}{\partial t}-c \frac{\partial v}{\partial x}=k(u, v, x)(u-v),
\end{array}\right.
$$

where $u=u(t, x), v=v(t, x), x \in \Omega \subseteq \mathbb{R}, t \in(0, T], T>0$, and $k(u, v, x)$ is a nonnegative function, called the interaction rate (also rate function or rate coefficient), that characterizes the interactions between gas particles. The model is in local equilibrium when $u=v$, a situation that will be obtained in the limit as we will see.

The case $k(u, v, x)=u+v$ was introduced by Carleman in the 1930's as a simplified model of the Boltzmann equation [1], and was subsequently studied by many authors (for a survey on these models see, for example, [7] and [8]).

\footnotetext{
*Dipartimento di Matematica, Università degli Studi di Pavia - Italy
} 
If we scale the variables by means of a small parameter $\varepsilon>0$ in such a way that the quotient $x^{2} / t$ is scaling invariant, one can easily show that System (1) is reduced to the form

$$
\left\{\begin{array}{l}
\frac{\partial u_{\varepsilon}}{\partial t}+\frac{1}{\varepsilon} \frac{\partial u_{\varepsilon}}{\partial x}=\frac{1}{\varepsilon^{2}} k\left(u_{\varepsilon}, v_{\varepsilon}, x\right)\left(v_{\varepsilon}-u_{\varepsilon}\right) \\
\frac{\partial v_{\varepsilon}}{\partial t}-\frac{1}{\varepsilon} \frac{\partial v_{\varepsilon}}{\partial x}=\frac{1}{\varepsilon^{2}} k\left(u_{\varepsilon}, v_{\varepsilon}, x\right)\left(u_{\varepsilon}-v_{\varepsilon}\right) \quad x \in \Omega \subseteq \mathbb{R}, t \geq 0 .
\end{array}\right.
$$

This scaling is particularly interesting because the limit $\varepsilon \rightarrow 0^{+}$(called the hydrodynamical limit), leads, at least formally, to diffusive type equations which can be viewed as the Navier-Stokes equations of the fictitious gas. Hence the name of diffusive scaling. Note that the new transport speed $1 / \varepsilon$ tends to infinity as $\varepsilon \rightarrow 0^{+}$.

The model is supplemented with initial data $u_{\varepsilon}(0, x)=u_{0}(x)$ and $v_{\varepsilon}(0, x)=v_{0}(x)$. Depending on the properties of the domain $\Omega$, also boundary conditions may be necessary.

The limiting process is as follows: we introduce two macroscopic variables, the mass density $\rho_{\varepsilon}$ and the flux $j_{\varepsilon}$ defined by

$$
\rho_{\varepsilon}=u_{\varepsilon}+v_{\varepsilon}, \quad j_{\varepsilon}=\frac{1}{\varepsilon}\left(u_{\varepsilon}-v_{\varepsilon}\right) .
$$

Typically, the rate has the form

$$
k_{\alpha}\left(u_{\varepsilon}, v_{\varepsilon}, x\right)=\left(u_{\varepsilon}+v_{\varepsilon}\right)^{\alpha} .
$$

In (4) $\alpha$ is a fixed constant ( $\alpha=1$ in Carleman's model). In this case, which is mostly considered in the literature, System (2) is equivalent to the following macroscopic equations for the mass density and the flux

$$
\left\{\begin{array}{l}
\frac{\partial \rho_{\varepsilon}}{\partial t}+\frac{\partial j_{\varepsilon}}{\partial x}=0 \\
\varepsilon^{2} \frac{\partial j_{\varepsilon}}{\partial t}+\frac{\partial \rho_{\varepsilon}}{\partial x}=-2 \rho_{\varepsilon}^{\alpha} j_{\varepsilon}
\end{array}\right.
$$

posed in $(t, x) \in(0, T] \times \Omega$ with initial data $\rho_{\varepsilon}(0, x)=u_{0}(x)+v_{0}(x)$ and $j_{\varepsilon}(0, x)=$ $\left[u_{0}(x)-v_{0}(x)\right] / \varepsilon$. If we are now allowed to disregard the term $\varepsilon^{2} \partial j_{\varepsilon} / \partial t$ in the limit $\varepsilon \rightarrow 0$, we formally arrive at the following nonlinear heat equation for the limit density $\rho=\lim _{\varepsilon \rightarrow 0} \rho_{\varepsilon}$ :

$$
\frac{\partial \rho}{\partial t}=\frac{1}{2} \frac{\partial}{\partial x}\left(\frac{1}{\rho^{\alpha}} \frac{\partial \rho}{\partial x}\right)
$$

with initial conditions $\rho(0, x)=u_{0}(x)+v_{0}(x)$. This is called the diffusive limit. Much more general forms of the rate function $k$ can be admitted in the study of this limit process (see [10] and its references). For such general $k$ the denominator $\rho^{\alpha}$ in the limit equation (6) becomes $k(\rho / 2, \rho / 2, x)$. However, we will consider in this paper only rates of the form $k_{\alpha}$ as given in (4).

The mathematical problem that we consider is to justify this limit process for different choices of $\alpha$ and under suitable assumptions on the data. The same goal has been 
the object of a number of papers, where information is given on the convergence of $\rho_{\varepsilon}=u_{\varepsilon}+v_{\varepsilon}$ as $\varepsilon \rightarrow 0^{+}$to a function $\rho(t, x)$ which solves the nonlinear diffusive process.

In particular, Lions and Toscani [4] solved the case $\alpha \in(-\infty, 1]$ with integrable data plus some regularity or decay conditions.

Subsequently, Salvarani and Vázquez [10] improved the result of [4], in the case $|\alpha| \leq 1$, by considering only $L^{1}$-data and studied the cases $\alpha>1$ with integrable data by using a special way of approximation (lifting approximation). They showed that, for $\alpha \geq 2$, the diffusive limit is trivial, since it reflects the nonexistence properties for the target equation, cf. [11]. On the other hand, for $1<\alpha<2$, while the limit should solve the expected diffusive problem with the same initial data, the mathematical properties of the differential operators are worse for $\alpha>1$ than for $|\alpha| \leq 1$, and the same strategy used in $[10]$ to prove convergence in the strong way of the space $C\left([0, T] ; L^{1}(\mathbb{R})\right)$ cannot be applied. A main problem is the failure of the maximum principle, which is heavily used in [10].

The object of this note is to show that in the subrange $1<\alpha<4 / 3$ compactness methods still apply under mild restrictions on the data and allow to prove that the semigroup generated by the hyperbolic problem still converges to the diffusive limit without any prior approximation of the data. Thus we extend the work done in [4] and [10] for $\alpha \leq 1$. We remark that the subrange analyzed in this work corresponds to consider equation (6) in the regime of very fast diffusion [12].

The method we use is based on the uniform control (with respect to $\varepsilon$ ) of a Lyapunov functional which is bounded from below only in the subrange $1<\alpha<4 / 3$. Thus, our method of proof can not be extended to values of the parameter $\alpha$ which are greater or equal than $4 / 3$.

To clarify the numerology which is behind this diffusive limit, in the second part of the paper we introduce a modified kinetic system that, while preserving all the mathematical properties of System (1), admits an explicit exact solution, which is shown to have a bounded support for all values of $\alpha$. The analysis of the behavior of this solution as $\varepsilon \rightarrow 0$ allows to enlighten the different properties of the diffusive limit in terms of the different values of the parameter $\alpha$.

To conclude this introduction, it is worth to mention that also the first initialboundary value problem for System (1), with non-homogeneous boundary conditions, has been investigated in [9], [3] by means of entropy methods. The main difference here is that, due to the presence of non-vanishing boundary conditions, the approach requires to use a relative entropy functional with respect to some suitable reference profile.

In more details, the main result of the paper is summarized in Theorem 4, Section 4. The proof requires various estimates which are the core of Section 3. Finally, Section 5 tries to give an answer to the problem of establishing a solid theory in the still remaining range $\alpha \in[4 / 3,2)$, by introducing a modified kinetic model. 


\section{Preliminaries}

This section collects some basic properties of System (2), that will be the starting point of the study about the diffusive limit of the model.

We will restrict to consider initial conditions which satisfy the properties listed in the following

Definition 1 Consider System (2) with $\alpha \in(1,4 / 3)$, posed for $(t, x) \in(0, T] \times \mathbb{R}$, with initial condition $u_{\varepsilon}(0, x)=u_{0}(x), v_{\varepsilon}(0, x)=v_{0}(x)$. The initial data $\left(u_{0}, v_{0}\right)$ are said to be admissible if and only if

1. $u_{0}$ and $v_{0}$ are nonnegative functions a.e. in $\mathbb{R}$ and are of class $L^{\infty}(\mathbb{R})$;

2. there exists a positive constant $\delta$ with $(\alpha-1) /(2(2-\alpha))<\delta<1 / 4$ such that

$$
\int_{\mathbb{R}}\left(u_{0}+v_{0}\right)\left(1+x^{2}\right)^{\delta} d x<C_{\delta}<\infty
$$

Remark It is immediate to see that the existence of $\delta$ requires that $(\alpha-1) /(2(2-\alpha))<$ $1 / 4$, which holds true exactly when $\alpha \in(1,4 / 3)$. We remark moreover that any admissible initial condition $\left(u_{0}, v_{0}\right)$ is of class $L^{1}(\mathbb{R}) \cap L^{\infty}(\mathbb{R})$. Indeed, we have that

$$
\left\|u_{0}+v_{0}\right\|_{L^{1}(\mathbb{R})} \leq \int_{\mathbb{R}}\left(u_{0}+v_{0}\right)\left(1+x^{2}\right)^{\delta} d x .
$$

An important consequence of the properties of the functions which are admissible initial data in the sense of Definition 1 is given by the following lemma:

Lemma 1 Let $\left(u_{0}, v_{0}\right)$ be admissible initial conditions for System (2), with $\alpha \in(1,4 / 3)$. Then the integral

$$
\int_{\mathbb{R}}\left(u_{0}^{2-\alpha}+v_{0}^{2-\alpha}\right) d x
$$

is finite.

Proof: We perform the computations only for $u_{0}$. We note that, for $\gamma>0$ :

$$
\int_{\mathbb{R}} u_{0}^{2-\alpha} d x \leq\left(\int_{\mathbb{R}} u_{0}\left(1+x^{2}\right)^{\gamma /(2-\alpha)} d x\right)^{2-\alpha}\left(\int_{\mathbb{R}}\left(1+x^{2}\right)^{\gamma /(1-\alpha)} d x\right)^{\alpha-1} .
$$

The first integral in the right-hand side of inequality (7) is finite, by definition of admissible initial data, by choosing $\gamma /(2-\alpha)=\delta<1 / 4$. Then, the last integral in the right-hand side of (7) is finite if $\gamma>(\alpha-1) / 2$. This requirement is satisfied whenever $\delta$ lies in $(\alpha-1) /(2(2-\alpha))<\delta<1 / 4$. Hence the thesis follows.

Next, we specify the sense of the solution we consider. We have: 
Definition $2 A$ weak solution of System (2) with admissible initial data $u_{0}, v_{0} \geq 0$ is a pair of functions $(u, v) \in C\left([0, T] ; L_{\mathrm{loc}}^{1}(\mathbb{R})\right) \cap L_{\mathrm{loc}}^{\infty}((0, T) \times \mathbb{R}), T>0$, such that the equation is satisfied in the sense of distributions and the initial data are recovered in the sense of traces.

By applying the strategy of Section 2 in [4], it is easy to obtain that the following property holds:

Proposition 1 Let $\left(u_{0}, v_{0}\right)$ admissible initial data for System (2).Then

$$
\left\|u_{\varepsilon}(t, \cdot)\right\|_{L^{p}(\mathbb{R})}^{2}+\left\|v_{\varepsilon}(t, \cdot)\right\|_{L^{p}(\mathbb{R})}^{2} \leq\left\|u_{0}\right\|_{L^{p}(\mathbb{R})}^{2}+\left\|v_{0}\right\|_{L^{p}(\mathbb{R})}^{2}
$$

for all $p$ such that $1<p \leq+\infty$ and for all $t>0$. Moreover,

$$
\left\|u_{\varepsilon}(t, \cdot)+v_{\varepsilon}(t, \cdot)\right\|_{L^{1}(\mathbb{R})}^{2}=\left\|u_{0}+v_{0}\right\|_{L^{1}(\mathbb{R})}^{2} \cdot
$$

The following theorem is proved in [10]:

Theorem 2 Let $0 \leq u_{0}(x), v_{0}(x) \in L_{\text {loc }}^{\infty}(\mathbb{R})$. Then the initial value problem for System $(2)$ admits a unique solution $u_{\varepsilon}(t, x), v_{\varepsilon}(t, x) \in C\left([0, \infty) ; L_{\mathrm{loc}}^{\infty}(\mathbb{R})\right)$. Moreover, this solution is non-negative.

\section{Control of the flux}

From now on, we will suppose always that $\alpha \in(1,4 / 3)$.

The core of the paper is the proof of a uniform estimate of the $L^{2}$-norm of the flux, which is based on the control of an entropy functional of System (2), defined as

$$
H_{\alpha}\left(u_{\varepsilon}, v_{\varepsilon}\right)=-\int_{\mathbb{R}}\left(u_{\varepsilon}^{2-\alpha}+v_{\varepsilon}^{2-\alpha}\right) d x .
$$

The following result holds:

Proposition 3 Let $\left(u_{\varepsilon}, v_{\varepsilon}\right)$ be a solution of the scaled discrete velocity system (2) with admissible initial data $\left(u_{0}, v_{0}\right)$. Then there exists a positive constant $Y \equiv Y\left(\alpha, u_{0}, v_{0}, T, \delta\right)>$ 0 such that the current $j_{\varepsilon}=\left(u_{\varepsilon}-v_{\varepsilon}\right) / \varepsilon$ satisfies

$$
\left\|j_{\varepsilon}^{2}\right\|_{L^{2}((0, T) \times \mathbb{R})} \leq Y
$$

for each $\varepsilon>0$. Moreover, there exists a positive constant $W \equiv W\left(\alpha, u_{0}, v_{0}, T, \delta\right)>0$ such that

$$
\int_{\mathbb{R}}\left(1+x^{2}\right)^{\delta}\left(u_{\varepsilon}+v_{\varepsilon}\right) d x \leq W .
$$

Proof: We consider the time evolution of the entropy. We obtain easily that

$$
\frac{d}{d t} H_{\alpha}\left(u_{\varepsilon}, v_{\varepsilon}\right)=-\frac{2-\alpha}{\varepsilon^{2}} \int_{\mathbb{R}} \rho_{\varepsilon}^{\alpha}\left(v_{\varepsilon}-u_{\varepsilon}\right)\left(u_{\varepsilon}^{1-\alpha}-v_{\varepsilon}^{1-\alpha}\right) d x \leq 0
$$


since $\left(v_{\varepsilon}-u_{\varepsilon}\right)\left(u_{\varepsilon}^{1-\alpha}-v_{\varepsilon}^{1-\alpha}\right) \geq 0$ for any $\alpha \in(1,2)$.

We observe now that, when $u_{\varepsilon}, v_{\varepsilon}>0$

$$
\rho_{\varepsilon}^{\alpha}\left(v_{\varepsilon}-u_{\varepsilon}\right)\left(u_{\varepsilon}^{1-\alpha}-v_{\varepsilon}^{1-\alpha}\right)=-\rho_{\varepsilon}^{\alpha}\left(u_{\varepsilon}-v_{\varepsilon}\right)^{2} \frac{u_{\varepsilon}^{1-\alpha}-v_{\varepsilon}^{1-\alpha}}{u_{\varepsilon}-v_{\varepsilon}}
$$

and by Lagrange's theorem there exists $\theta \in[0,1]$ such that

$$
\begin{gathered}
-\rho_{\varepsilon}^{\alpha}\left(u_{\varepsilon}-v_{\varepsilon}\right)^{2} \frac{u_{\varepsilon}^{1-\alpha}-v_{\varepsilon}^{1-\alpha}}{u_{\varepsilon}-v_{\varepsilon}}= \\
(\alpha-1)\left(u_{\varepsilon}-v_{\varepsilon}\right)^{2} \frac{\left(u_{\varepsilon}+v_{\varepsilon}\right)^{\alpha}}{\left(\theta u_{\varepsilon}+(1-\theta) v_{\varepsilon}\right)^{\alpha}} \geq(\alpha-1)\left(u_{\varepsilon}-v_{\varepsilon}\right)^{2} .
\end{gathered}
$$

When $u_{\varepsilon}$ and/or $v_{\varepsilon}=0$, an estimate of the time derivative of the entropy through the current is immediate.

We have hence deduced that

$$
\frac{d}{d t} H_{\alpha}\left(u_{\varepsilon}, v_{\varepsilon}\right) \leq-(2-\alpha)(\alpha-1) \int_{\mathbb{R}} j_{\varepsilon}^{2} d x
$$

for all non-negative solution $\left(u_{\varepsilon}, v_{\varepsilon}\right)$ of System (2).

We now need to give an estimate of the mass which is lost at infinity, which we obtain by controlling some moment of the density.

We introduce therefore a parameter $\delta \in(0,1 / 4)$ and study the time evolution of the moment of order $2 \delta$ :

$$
\frac{d}{d t} \int_{\mathbb{R}}\left(1+x^{2}\right)^{\delta}\left(u_{\varepsilon}+v_{\varepsilon}\right) d x=-\int_{\mathbb{R}}\left(1+x^{2}\right)^{\delta} \frac{\partial j_{\varepsilon}}{\partial x} d x=2 \delta \int_{\mathbb{R}} x\left(1+x^{2}\right)^{\delta-1} j_{\varepsilon} d x .
$$

Then, by using the elementary inequality $2 a b \leq c a^{2}+b^{2} / c, c>0$, we have

$$
2 \delta \int_{\mathbb{R}} \frac{x}{\left(1+x^{2}\right)^{1-\delta}} j_{\varepsilon} d x \leq c \int_{\mathbb{R}} j_{\varepsilon}^{2} d x+\frac{\delta^{2}}{c} \int_{\mathbb{R}} \frac{x^{2}}{\left(1+x^{2}\right)^{2-2 \delta}} d x
$$

Since $\delta \in(0,1 / 4)$, then the integral

$$
I_{\delta}=\int_{\mathbb{R}} \frac{x^{2}}{\left(1+x^{2}\right)^{2-2 \delta}} d x<+\infty
$$

and hence, we can conclude, by choosing $c=(2-\alpha)(\alpha-1) / 2$, that

$$
\frac{d}{d t} \int_{\mathbb{R}}\left[\left(1+x^{2}\right)^{\delta}\left(u_{\varepsilon}+v_{\varepsilon}\right)+H_{\alpha}\left(u_{\varepsilon}, v_{\varepsilon}\right)\right] d x \leq \frac{1}{2}(2-\alpha)(1-\alpha) \int_{\mathbb{R}} j_{\varepsilon}^{2} d x+\frac{2 \delta^{2}}{(2-\alpha)(\alpha-1)} I_{\delta}
$$

We then integrate the previous inequality with respect to $t$ in $(0, T)$, with $T>0$. We deduce that

$$
\mathcal{H}\left(u_{\varepsilon}, v_{\varepsilon}\right) \leq \mathcal{H}\left(u_{0}, v_{0}\right)+\frac{2 T \delta^{2}}{(2-\alpha)(\alpha-1)} I_{\delta}
$$


where

$$
\mathcal{H}(t)=\mathcal{H}\left(u_{\varepsilon}, v_{\varepsilon}\right)=\int_{\mathbb{R}}\left[\left(1+x^{2}\right)^{\delta}\left(u_{\varepsilon}+v_{\varepsilon}\right)+H_{\alpha}\left(u_{\varepsilon}, v_{\varepsilon}\right)\right] d x .
$$

We observe that

$$
\mathcal{H}\left(u_{0}, v_{0}\right)=\int_{\mathbb{R}}\left[\left(1+x^{2}\right)^{\delta}\left(u_{0}+v_{0}\right)+H_{\alpha}\left(u_{0}, v_{0}\right)\right] d x<+\infty
$$

because the data are admissible.

Moreover,

$$
\begin{gathered}
\mathcal{H}(t) \geq \int_{\mathbb{R}}\left(1+x^{2}\right)^{\delta} u_{\varepsilon} d x-\left(\int_{\mathbb{R}}\left(1+x^{2}\right)^{\delta} u_{\varepsilon} d x\right)^{2-\alpha} K_{\delta}^{\alpha-1}+ \\
\int_{\mathbb{R}}\left(1+x^{2}\right)^{\delta} v_{\varepsilon} d x-\left(\int_{\mathbb{R}}\left(1+x^{2}\right)^{\delta} v_{\varepsilon} d x\right)^{2-\alpha} K_{\delta}^{\alpha-1}
\end{gathered}
$$

where

$$
K_{\delta}=\int_{\mathbb{R}}\left(1+x^{2}\right)^{\delta(2-\alpha) /(1-\alpha)} d x>0 .
$$

It is straightforward to see that, for any $\alpha \in(1,4 / 3)$, there exists $\delta \in(0,1 / 4)$ such that $K_{\delta}$ is finite.

Hence, we can deduce that there exists $\eta \in \mathbb{R}$ (non necessarily positive) such that $\mathcal{H}(t) \geq \eta$ for all $t \geq 0$ : indeed, we have that $\eta=2 m$, where $m$ is the (unique) minimum for the function $f(y)=y-y^{2-\alpha} K_{\delta}^{\alpha-1}$, for $y \geq 0$, that is

$$
m=\left[(2-\alpha)^{1 /(\alpha-1)}-(2-\alpha)^{(2-\alpha) /(\alpha-1)}\right] K_{\delta} .
$$

We hence deduce a posteriori that

$$
\frac{1}{2}(2-\alpha)(\alpha-1)\left\|j_{\varepsilon}\right\|_{L^{2}((0, T) \times \mathbb{R})}^{2} \leq \frac{2 T \delta^{2}}{\alpha-1} I_{\delta}+\mathcal{H}(0)-\mathcal{H}(T),
$$

that gives a bound (uniform with respect to $\varepsilon$ ) for the $L^{2}$-norm of the flux $j_{\varepsilon}$.

Finally, we use Cauchy-Schwarz inequality to obtain that

$$
\int_{0}^{T} \int_{\mathbb{R}} x\left(1+x^{2}\right)^{\delta-1} j_{\varepsilon} d x d t \leq\left\|j_{\varepsilon}^{2}\right\|_{L^{2}((0, T) \times \mathbb{R})}^{1 / 2}\left(\int_{0}^{T} \int_{\mathbb{R}} x^{2}\left(1+x^{2}\right)^{2 \delta-2} d x d t\right)^{1 / 2} .
$$

Since $\delta \in(0,1 / 4)$ and $j_{\varepsilon}$ is bounded in $L^{2}((0, T) \times \mathbb{R})$, the right-hand side of the previous inequality is bounded; we hence obtain from Equation (10) a bound (uniform with respect to $\varepsilon$ ) for the moment of order $2 \delta$. 


\section{The nonlinear diffusion limit}

The strategy of passing to the limit is classical. In the sequel, we will use various relatively compact sequences. When we will say that a sequence converges to a limit, we will mean that there exists a subsequence which converges to the limit.

The proof uses the bounds of Propositions 1 and 3 in order to deduce some properties of compactness.

Thanks to Proposition 1, by Banach-Alaoglu theorem, there exists $\rho \in L^{\infty}\left((0, T) ; L^{2}(\mathbb{R})\right)$ such that $\rho_{\varepsilon} \rightarrow \rho$ in $L^{\infty}\left((0, T) ; L^{2}(\mathbb{R})\right)$ weak*

Moreover, thanks to Proposition 3, again by Banach-Alaoglu theorem, there exists $j \in L^{2}((0, T) \times \mathbb{R})$ such that $j_{\varepsilon} \rightarrow j$ in $L^{2}((0, T) \times \mathbb{R})$.

Note that, since $\alpha>1$,

$$
\left\|\rho_{\varepsilon}^{\alpha} j_{\varepsilon}\right\|_{L^{2}((0, T) \times \mathbb{R})}^{2} \leq\left\|\rho_{\varepsilon}(t, \cdot)\right\|_{L^{\infty}(\mathbb{R})}^{2 \alpha}\left\|j_{\varepsilon}\right\|_{L^{2}((0, T) \times \mathbb{R})}^{2} \leq\left\|u_{0}+v_{0}\right\|_{L^{\infty}(\mathbb{R})}^{2 \alpha}\left\|j_{\varepsilon}\right\|_{L^{2}((0, T) \times \mathbb{R})}^{2} .
$$

Since the problem is nonlinear, weak convergence is not enough to pass to the limit; strong convergence is needed. We will obtain it by using a well knonw result of compensated compactness. Consider the vector fields

$$
p_{\varepsilon}=\left(\rho_{\varepsilon}, j_{\varepsilon}\right) \text { and } q_{\varepsilon}=\left(-\rho_{\varepsilon}, \varepsilon^{2} j_{\varepsilon}\right) .
$$

By Propositions 1 and 3, both vector fields satisfy

$$
p_{\varepsilon} \text { and } q_{\varepsilon} \text { are bounded in } L^{2}\left([0, T] \times \mathbb{R} ; \mathbb{R}^{2}\right) .
$$

Summing the first two equations in the scaled generalized Carleman system (2) shows that

while

$$
\operatorname{div}_{t, x} p_{\varepsilon}=\frac{\partial \rho_{\varepsilon}}{\partial t}+\frac{\partial j_{\varepsilon}}{\partial x}=0
$$

$$
\operatorname{curl}_{t, x} q_{\varepsilon}=\varepsilon^{2} \frac{\partial j_{\varepsilon}}{\partial t}+\frac{\partial \rho_{\varepsilon}}{\partial x}=-2 \rho_{\varepsilon}^{\alpha} j_{\varepsilon}
$$

We then obtain that

$$
\operatorname{div}_{t, x} p_{\varepsilon} \text { and } \operatorname{curl}_{t, x} q_{\varepsilon} \text { bounded in } L^{2}([0, T] \times \mathbb{R}) .
$$

By compensated compactness (the div-curl lemma in [6]), we find that

$$
p_{\varepsilon} \cdot q_{\varepsilon}=-\rho_{\varepsilon}^{2}+\varepsilon^{2} j_{\varepsilon}^{2} \rightarrow p \cdot q=\rho^{2}
$$

in the sense of distributions on $(0, T) \times \mathbb{R}$ as $\varepsilon \rightarrow 0$. Because of Proposition 3 , this implies that $\rho_{\varepsilon}^{2} \rightarrow \rho^{2}$ in the sense of distributions, which implies in turn that the family $\rho_{\varepsilon}$ is relatively compact in $L^{2}([0, T] \times \mathbb{R})$ strong thanks to the argument of [5], Section 4 .

Therefore we can pass to the limit in System (5). However, it is known [2] that the target equation (6) does not have uniqueness of solutions in the range $\alpha \in[1,2)$. By proposition 1, we can characterize our limit as the unique maximal solution of Equation (6), which conserves the mass.

We have hence proved the following result: 
Theorem 4 Let $\left(u_{0}, v_{0}\right)$ be admissible data for the generalized Carleman system. For each $\varepsilon>0$, let $\left(u_{\varepsilon}, v_{\varepsilon}\right)$ be the solution of the scaled Carleman system (2) with admissible initial condition and let $\alpha \in(1,4 / 3)$.

Then, in the limit as $\varepsilon \rightarrow 0$, the macroscopic density

$$
\rho_{\varepsilon}=u_{\varepsilon}+v_{\varepsilon} \rightarrow \rho \quad \text { in } L_{\mathrm{loc}}^{p}((0, T) \times \mathbb{R})
$$

for all $p \in(1,+\infty)$ where $\rho$ is a generalized solution of (6) when $\alpha \in(1,4 / 3)$, with initial conditions $\rho(0, x)=u_{0}(x)+v_{0}(x)$.

We note that the previous theorem gives also a way to prove the existence of a solution for the target equation (6) - something which could be proven in a simpler way by using the standard theory on fast diffusion equations.

\section{A semiformal approach to numerology}

The method of the previous Sections, which allows to prove our main Theorem 4 , requires that $\alpha \in(1,4 / 3)$. Indeed, the control on the $L^{2}$-norm of the flux obtained in Proposition 3 depends on the entropy functional (8), which is bounded from below only in this range of the parameter $\alpha$.

In addition, in the remaining range $\alpha \in[4 / 3,2)$, it is known that the maximal solution of the target equation $\rho$, which can be uniquely characterized by the conservation of the mass, has a behavior, when $|x| \rightarrow+\infty$, which does not allow to deduce that the integral

$$
\int_{\mathbb{R}} \rho^{2-\alpha}(t, x) d x
$$

is finite [2]. Hence, the lack of control on the moments of the solution of the target nonlinear diffusion makes our argument, which is based on the time evolution of the entropy functional (8), inapplicable in that range.

On the other hand, while our method of proof cannot be extended to cover the remaining unstudied range $\alpha \in[4 / 3,2)$, there are no essential obstructions to prove a similar result by using different techniques. Like it happens in the target hydrodynamic equation, which is a nonlinear diffusion, the knowledge of an exact solution would certainly help to understand the limiting process. Unfortunately, despite the famous Carleman model, where existence of self-similar solutions has been discovered many years ago [8], when $\alpha \neq 1$ System (2) does not exhibit self-similar profiles for the densities.

A simple modification of System (2), however, allows to obtain exact solutions, without affecting both the properties of the solution at a kinetic level, and the convergence proofs to hydrodynamics, when available.

Let us multiply the collisional part of System (2) by the function of time $\sigma(t)=$ $(1+t)^{\alpha-1}$. We obtain the new system

$$
\left\{\begin{array}{c}
\frac{\partial u_{\varepsilon}}{\partial t}+\frac{1}{\varepsilon} \frac{\partial u_{\varepsilon}}{\partial x}=\frac{\sigma(t)}{\varepsilon^{2}}\left(u_{\varepsilon}+v_{\varepsilon}\right)^{\alpha}\left(v_{\varepsilon}-u_{\varepsilon}\right) \\
\frac{\partial v_{\varepsilon}}{\partial t}-\frac{1}{\varepsilon} \frac{\partial v_{\varepsilon}}{\partial x}=\frac{\sigma(t)}{\varepsilon^{2}}\left(u_{\varepsilon}+v_{\varepsilon}\right)^{\alpha}\left(u_{\varepsilon}-v_{\varepsilon}\right)
\end{array}\right.
$$


where $x \in \Omega \subseteq \mathbb{R}, t \geq 0$. We remark that, for Carleman model, $\alpha=1$ : hence $\sigma(t)=1$ and System (11) coincides with (2).

Moreover, since in a bounded interval of time, $t \leq T$, the function $\sigma(t)$ remains uniformly bounded both from below and above, the existing results on System (2), $\alpha \leq 4 / 3$, remain valid also for System (11).

The corresponding macroscopic equations for the mass density and the flux now read

$$
\left\{\begin{array}{l}
\frac{\partial \rho_{\varepsilon}}{\partial t}+\frac{\partial j_{\varepsilon}}{\partial x}=0 \\
\varepsilon^{2} \frac{\partial j_{\varepsilon}}{\partial t}+\frac{\partial \rho_{\varepsilon}}{\partial x}=-2 \sigma(t) \rho_{\varepsilon}^{\alpha} j_{\varepsilon}
\end{array}\right.
$$

As before, equations (12) are posed in $(t, x) \in(0, T] \times \mathbb{R}$ with initial data $\rho_{\varepsilon}(0, x)=$ $u_{0}(x)+v_{0}(x)$ and $j_{\varepsilon}(0, x)=\left[u_{0}(x)-v_{0}(x)\right] / \varepsilon$. Again, if we are allowed to disregard the term $\varepsilon^{2} \partial j_{\varepsilon} / \partial t$ in the limit $\varepsilon \rightarrow 0$, we obtain that the limit density $\rho=\lim _{\varepsilon \rightarrow 0} \rho_{\varepsilon}$ satisfies the nonlinear diffusion

$$
\frac{\partial \rho}{\partial t}=\frac{1}{2 \sigma(t)} \frac{\partial}{\partial x}\left(\frac{1}{\rho^{\alpha}} \frac{\partial \rho}{\partial x}\right)
$$

with initial conditions $\rho(0, x)=u_{0}(x)+v_{0}(x)$. Hence, the modified System (11) has a diffusive limit which is nothing but the diffusive limit of System (2), suitably scaled in time. In fact, if we now rescale the time in such a way that $\tau^{\prime}(t)=\sigma^{-1}(t)$, the new unknown $\bar{\rho}(\tau, x)=\rho(t, x)$ satisfies

$$
\frac{\partial \bar{\rho}}{\partial \tau}=\frac{1}{2} \frac{\partial}{\partial x}\left(\frac{1}{\bar{\rho}^{\alpha}} \frac{\partial \bar{\rho}}{\partial x}\right)
$$

namely equation (6). Note that the time rescaling $\tau^{\prime}(t)=\sigma^{-1}(t)$ implies that the new time $\tau(t)$ is

$$
\tau(t)=\frac{1}{(2-\alpha)}(1+t)^{2-\alpha}+\text { const. }
$$

This scaling does not change the time direction only for $\alpha<2$. This is another way to show that a meaningful diffusive limit can be obtained only when the parameter $\alpha$ is strictly less than 2 .

One of the advantages of working with System (11) is that, similarly to Carleman's system, we can look for self-similar profiles. To this aim, for given functions of time $\gamma(t)$ and $\tau(t)$ let us define new densities $(\mathcal{U}, \mathcal{V})$ by the position

$$
u_{\varepsilon}(t, x)=\frac{1}{\gamma(t)} \mathcal{U}\left(\tau(t), \frac{x}{\gamma(t)}\right) ; \quad v_{\varepsilon}(t, x)=\frac{1}{\gamma(t)} \mathcal{V}\left(\tau(t), \frac{x}{\gamma(t)}\right)
$$

It is only matter of simple computations to show that, taking $\gamma(t)=1+t$ and $\tau(t)=$ $\log \gamma(t)$ the pair $(\mathcal{U}(\tau, y), \mathcal{V}(\tau, y))$ satisfies the system

$$
\left\{\begin{array}{l}
\frac{\partial \mathcal{U}}{\partial \tau}+\frac{1}{\varepsilon} \frac{\partial \mathcal{U}}{\partial y}=\frac{\sigma(t)}{\varepsilon^{2}}(\mathcal{U}+\mathcal{V})^{\alpha}(\mathcal{V}-\mathcal{U})+\frac{\partial}{\partial y}(y \mathcal{U}) \\
\frac{\partial \mathcal{V}}{\partial t}-\frac{1}{\varepsilon} \frac{\partial \mathcal{V}}{\partial y}=\frac{\sigma(t)}{\varepsilon^{2}}(\mathcal{U}+\mathcal{V})^{\alpha}(\mathcal{U}-\mathcal{V})+\frac{\partial}{\partial y}(y \mathcal{V})
\end{array}\right.
$$


Note that, since $\gamma(0)=1, \tau(0)=0$ and the new initial densities $(\mathcal{U}(0, y(0)), \mathcal{V}(0, y(0)))$ coincide with the old ones $\left(u_{0}(x), v_{0}(x)\right)$.

As usual in these cases, let us look for stationary solutions $(\mathcal{U}(y), \mathcal{V}(y))$ of System (15). Let us work in terms of the macroscopic quantities

$$
R(y)=\mathcal{U}(y)+\mathcal{V}(y), \quad J(y)=\frac{1}{\varepsilon}(\mathcal{U}(y)-\mathcal{V}(y)) .
$$

The pair $(R, J)$ of stationary mass and flux satisfies the system

$$
\left\{\begin{array}{l}
\frac{d J}{d y}=\frac{d}{d y}(y R) \\
\frac{1}{\varepsilon} \frac{d R}{d y}=-\frac{2}{\varepsilon} R^{\alpha} J+\varepsilon \frac{d}{d y}(y J) .
\end{array}\right.
$$

The first equation implies that $J=y R+c, c \in \mathbb{R}$. Let us choose the constant $c=0$. By substituting $J=x R$ in the second equation, we obtain an ordinary differential equation for the unknown $R$

$$
\frac{d}{d y}\left[\left(1-\varepsilon^{2} y^{2}\right) R\right]=-2 y R^{\alpha+1} .
$$

It can be easily seen that the positivity of the solution requires $|\varepsilon y|<1$. By assuming $R=0$ in the remaining set $|\varepsilon y| \geq 1$, and defining

$$
G(y)=\left(1-\varepsilon^{2} y^{2}\right) R(y), \quad|\varepsilon y|<1,
$$

we obtain that $G$ satisfies

$$
\frac{1}{G^{\alpha+1}} \frac{d G}{d y}=-\frac{2 y}{\left(1-\varepsilon^{2} y^{2}\right)^{\alpha+1}},
$$

which can be easily solved integrating from 0 to $y$ to give, for $\alpha>0$

$$
G(y)=\left[A+\frac{1}{\varepsilon^{2}}\left(\left(1-\varepsilon^{2} y^{2}\right)^{-\alpha}-1\right)\right]^{-1 / \alpha} .
$$

Connecting things, and denoting by $\chi_{(E)}$ the characteristic function of the set $E \subseteq \mathbb{R}$, we obtain that, for $\alpha>0$ the steady mass is

$$
R_{\infty}^{\varepsilon}(y)=\left[A\left(1-\varepsilon^{2} y^{2}\right)^{\alpha}+\frac{1}{\varepsilon^{2}}\left(1-\left(1-\varepsilon^{2} y^{2}\right)^{\alpha}\right)\right]^{-1 / \alpha} \chi_{(|y|<1 / \varepsilon)}
$$

while the flux reads

$$
J_{\infty}^{\varepsilon}(y)=y\left[A\left(1-\varepsilon^{2} y^{2}\right)^{\alpha}+\frac{1}{\varepsilon^{2}}\left(1-\left(1-\varepsilon^{2} y^{2}\right)^{\alpha}\right)\right]^{-1 / \alpha} \chi_{(|y|<1 / \varepsilon)},
$$

We remark that the constant $A$ has to be chosen in such a way that $\left\|R_{\infty}^{\varepsilon}\right\|_{L^{1}(\mathbb{R})}=1$. 
Going back to the original variables, we found for System (11) the exact solutions

$$
\begin{aligned}
& \rho_{\infty}^{\varepsilon}(t, x)=\frac{1}{1+t}\left[\frac{1}{\varepsilon^{2}}+\left(A-\frac{1}{\varepsilon^{2}}\right)\left(1-\left(\frac{\varepsilon x}{1+t}\right)^{2}\right)^{\alpha}\right]^{-1 / \alpha} \chi_{(|x|<(1+t) / \varepsilon),} \\
& j_{\infty}^{\varepsilon}(t, x)=\frac{x}{(1+t)^{2}}\left[\frac{1}{\varepsilon^{2}}+\left(A-\frac{1}{\varepsilon^{2}}\right)\left(1-\left(\frac{\varepsilon x}{1+t}\right)^{2}\right)^{\alpha}\right]^{-1 / \alpha} \chi_{(|x|<(1+t) / \varepsilon)} .
\end{aligned}
$$

The pair $\left(\rho_{\infty}^{\varepsilon}(t, x), j_{\infty}^{\varepsilon}(t, x)\right)$ of exact solutions is compactly supported, and the support growths at the rate $(1+t) / \varepsilon$. It is remarkable that these solutions have a jump at the extremal points of the support. In fact, while $\rho_{\infty}^{\varepsilon}(t, x)=0$ for $x \geq(1+t) / \varepsilon$,

$$
\lim _{x \rightarrow[(1+t) / \varepsilon]^{-}} \rho_{\infty}^{\varepsilon}(t, x)=\frac{\varepsilon^{2 / \alpha}}{1+t} .
$$

Thus, for any fixed $\varepsilon$ the solution represents a shock-like solution expanding with time. Due to the fact that this solution has bounded support, however, for a fixed value of the scaling parameter $\varepsilon$, all moments stay bounded. Analogously, the flux $j_{\infty}^{\varepsilon}$ is bounded in any $L^{p}$-space, $p \geq 1$.

On the other hand, as $\varepsilon \rightarrow 0, \rho_{\infty}^{\varepsilon}(t, x)$ converges to the Barenblatt-like profile

$$
\rho_{\infty}(t, x)=\frac{1}{1+t}\left(A+\frac{\alpha x^{2}}{(1+t)^{2}}\right)^{-1 / \alpha}
$$

which has unbounded support, and only a finite number of moments bounded. In particular, the uniform $L^{2}$-boundedness of the flux $\left.j_{\infty}^{\varepsilon}(t, x)\right)$ is lost in the limit as soon as the function

$$
j_{\infty}(t, x)=\frac{x}{(1+t)^{2}}\left(A+\frac{\alpha x^{2}}{(1+t)^{2}}\right)^{-1 / \alpha}
$$

does not belong to $L^{2}(\mathbb{R})$. Clearly this happens when $\alpha \geq 4 / 3$. To go over this barrier we need to control the flux in $L^{p}(\mathbb{R})$, with $p>2$. This can not be done through the entropy production, and the entropy strategy fails.

It is also interesting to remark that if $\alpha<0$, the solution $G$ to equation (16) satisfies

$$
G(y)^{-\alpha}=A-\frac{1}{\varepsilon^{2}}\left(1-\left(1-\varepsilon^{2} y^{2}\right)^{-\alpha}\right),
$$

and positivity is guaranteed only if

$$
y^{2} \leq \frac{1-\left(1-A \varepsilon^{2}\right)^{-1 / \alpha}}{\varepsilon^{2}} .
$$

In this case the steady solution of the form

$$
R_{\infty}^{\varepsilon}(y)=\frac{\left[\frac{1}{\varepsilon^{2}}\left(1-\varepsilon^{2} y^{2}\right)^{-\alpha}+\left(A-\frac{1}{\varepsilon^{2}}\right)\right]^{-1 / \alpha} \chi_{\left(|y|<C_{\varepsilon}^{\alpha}\right)}}{1-\varepsilon^{2} y^{2}}
$$


where

$$
C_{\varepsilon}^{\alpha}=\min \left\{1 / \varepsilon, \frac{\left(1-\left(1-A \varepsilon^{2}\right)^{-1 / \alpha}\right)^{1 / 2}}{\varepsilon}\right\} .
$$

It turns out that, for $\varepsilon$ sufficiently small, $\varepsilon \leq \varepsilon_{\alpha}$,

$$
C_{\varepsilon}^{\alpha}=\frac{\left(1-\left(1-A \varepsilon^{2}\right)^{-1 / \alpha}\right)^{1 / 2}}{\varepsilon} \leq C<+\infty .
$$

In this case the steady solution is continuous on the whole real line, while the support stays bounded as $\varepsilon$ converges to 0 . From this we recognize the finite speed of spreading of the support for the mass $\rho_{\infty}(t, x)$.

Acknowledgment: The authors thank Prof J. L. Vázquez for fruitful discussions and the constant encouragement in writing the paper. Partial support of the Italian MIUR project "Kinetic and hydrodynamic equations of complex collisional systems" is kindly acknowledged.

\section{References}

[1] T. Carleman. Problèmes mathématiques dans la théorie cinétique des gaz, Almqvist-Wiksells, Uppsala, 1957.

[2] J. R. Esteban, A. Rodríguez, J. L. VÁzquez. A nonlinear heat equation with singular diffusivity, Comm. Partial Differential Equations 13 (1988), 985-1039.

[3] F. Golse, F. Salvarani. The nonlinear diffusion limit for generalized Carleman models: the initial-boundary value problem, Nonlinearity 2020 (2007), 927-942.

[4] P. L. Lions, G. Toscani. Diffusive limits for finite velocities Boltzmann kinetic models, Rev. Mat. Iberoamericana 13 (1997), 473-513.

[5] P. Marcati, A. J. Milani. The one-dimensional Darcy's law as the limit of a compressible Euler flow. J. Diff. Eq. 84 (1990), 129-147.

[6] F. Murat. Compacité par compensation, Ann. Scuola Norm. Sup. Pisa Cl. Sci. 5 (1978), 489-507.

[7] R. Monaco, L. Preziosi. Fluid Dynamic Applications of the Discrete Boltzmann Equation, World Scientific Publishing Co., River Edge, NJ, 1991.

[8] T. PŁatkowski, R. Illner. Discrete velocity models of the Boltzmann equation: a survey on the mathematical aspects of the theory, SIAM Review 30 (1988), 213255.

[9] F. Salvarani. Diffusion limits for the initial-boundary value problem of the Goldstein-Taylor model. Rend. Sem. Mat. Univ. Politec. Torino 57 (1999), 209-220. 
[10] F. Salvarani, J. L. VÁzquEz. The diffusive limit for Carleman-type kinetic models, Nonlinearity 18 (2005), 1223-1248.

[11] J. L. VÁzquez. Nonexistence of solutions for nonlinear heat equations of fastdiffusion type, J. Math. Pures Appl. 71 (1992), 503-526.

[12] J. L. VÁzquez. Smoothing and decay estimates for nonlinear diffusion equations, Vol. 33 of Oxford Lecture Notes in Maths. and its Applications, Oxford Univ. Press, Oxford 2006. 